\title{
Computational Genomics of RELA gene of Pig and its protein prescription
}

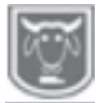

Abstract
Oluwole, O. O.

Institute of Agricultural Research and Training,

Obafemi Awolowo University, Nigeria

oluwafunmike@yahoo.co.uk+234(0)7031567164

RELA gene is a pleiotropic transcription that is present in almost all living cells where several signal transduction events end points were initiated by many arrays of stimuli that were related to many biological processes such as inflammation, immunity, differentiation, cell growth, tumorigenesis and apoptosis in the cells. The nucleotide and amino acid sequence (AAS) of RELA gene of pig and other mammalian species were downloaded from the National Center for Biotechnology information (NCBI) data base, United State of America and Universal protein resources (Uniprot) database, United Kingdom. Data generated were analyzed by bioinformatics tools. The results showed a high variation of AAS among the mammals where only four mammals have the same AAS length (551) with pig while others were not. The RELA gene of pig shared very high percent identity with all mammals ranging from $95.5 \%$ (warthog) to $83.5 \%$ (guinea pig) and similarity percentage ranged from 85.1 to $99.5 \%$ where the warthog had the highest percentage value while the least mammal was Guinea pig. The tree showed RELA gene with two clades; the first for mammals and the second for non-mammals. The RELA gene of pig was in the same cluster with the other Suidae family, warthog and babirusa. For the protein structure obtained, only $43 \%$ can be meaningfully predicted from the $61 \%$ residues modelled at more than $90 \%$. The domain architecture of RELA protein structure contained mainly RNA Recognition motif and region of low complexity. The domainstarting position of all the organisms ranged from 191 to 210 while the termination of all the mammals ranged from 102 to 306 amino acids. The region of low complexity starting point ranged from position 173 to 462 while termination positions ranged from 184 to 474.

The evolution relationship of RELA gene of pig and other mammalian species were highly related and conserved among the mammalian species; they have high comparability and they evolved from common ancestors.

Keywords: Pig, RELA gene, protein structure, phylogenetic tree.

\section{Introduction}

The RELA gene is a gene that encodes a protein called Transcription factor P65 known as Nuclear Factor NF-Kappa-B $\left(\mathrm{NF}_{\mathrm{k}} \mathrm{B}\right) \mathrm{P} 65$. It is involved in formation, nuclear translocation and activation of NF${ }_{\mathrm{k}} \mathrm{B}$ heterodimer (Nolan et al., 1991). It is essential because it is involved in all types of cellular processes such as cellular metabolism, chemotaxis etc. The major function of RELA is the phosphorylation and acetylation that are crucial for posttranslational modifications that are required for activation of NF- $\mathrm{NB}$. It also modulate immune responses and its activation is positively associated with multiple types of cancer in humans.

The NF-kappa-B complexes are held in the cytoplasm in an inactive state complexed with members of the NF-kappa-B inhibitor (I-kappa-B) family. In a conventional activation pathway, I-kappa-B is phosphorylated by I-kappa-B kinases (IKKs) in response to different activators, subsequently degraded thus liberating the active NF-kappa-B complex which 


\section{Computational Genomics of RELA gene of Pig and its protein prescription}

translocated to the nucleus. The NF-kappaB p65-p65 complex appears to be involved in invasin-mediated activation of IL-8 expression (http:// www. uniprot .org/ uniprot/Q04206). The inhibitory effect of Ikappa-B upon NF-kappa-B the cytoplasm is exerted primarily through the interaction with p65. The p65 shows a weak DNAbinding site which could contribute directly to DNA binding in the NF-kappa-B complex. It associates with chromatin at the NF-kappa-B promoter region via a s s c i a tion with D D X 1 (http://www.genecards.org/cgibin/carddisp.pl?gene=RELA). It is also essential for cytokine gene expression in Tcells (Bettelli et al., 2005).

As a complex compound with other protein, it contains a N-terminal REL-homology domain (RHD), and also a C-terminal transactivation domain (TAD), where RHD is involved in DNA binding, dimerization and NF- $\mathrm{KB} / \mathrm{REL}$ inhibitor interaction. On the other hand, TAD is responsible for interacting with the basal transcription complex (Chen and Greene, 2004). RELA and p50 is the mostly commonly found heterodimer complex among NF- $\mathrm{KB}$ homodimers and heterodimers, and is the functional component participating in nuclear translocation and activation of NFкB ((http://www.genecards.org/cgibin/carddisp.pl?gene=RELA).).

The RELA participates in adaptive immunity and responses to invading pathogens via NF- $\mathrm{KB}$ activation. Mice without individual NF- $\kappa \mathrm{B}$ proteins are deficient in B- and T-cell activation and proliferation, cytoline production and isotype switching (Li and Verma, 2002). He also reported that mutations in RELA are found responsible for inflammatory bowel disease as well. Therefore, this study was undertaken to evaluate the phylogenetic relationship between RELA gene of pig and other mammalian species and to predict its protein structure and its domain architecture.

\section{Materials and methods \\ Retrieval of amino acids sequences}

The nucleotide and amino acid sequences (AAS) of RELA gene of pig were downloaded from the National Center for Biotechnology information (NCBI) data base, United State of America and Universal protein resources (Uniprot) database, United Kingdom.

Determination of Percentage Identity and Similarity

The Identity and Similarity percentage of AAS of RELA gene of pig and other mammals were identified by conducting pairwise comparison of their AAS using two or more sequences of Basic local alignment search tool (BLAST).

\section{Phylogenetic analysis}

The phylogenetic tree was drawn to scale, with branch lengths in the same units as those of the evolutionary distances used to infer the phylogenetic tree. The evolutionary history was inferred by using the Maximum Likelihood method based on the JTT matrix-based model (Saitout and Nei, 1987). The tree with the highest $\log$ likelihood (-1182.1917) is shown. The percentage of trees in which the associated taxa clustered together is shown next to the branches. Initial tree(s) for the heuristic search were obtained automatically by applying Neighbor-Join and BioNJ algorithms to a matrix of pairwise distances estimated using a JTT model, and then selecting the topology with superior $\log$ likelihood value. The tree is drawn to scale, with branch lengths measured in the number of substitutions per site. The analysis involved 25 amino acid sequences. All positions containing gaps and missing data were eliminated. There were a total of 


\section{Oluwole}

171 positions in the final dataset. Evolutionary analyses were conducted in MEGA7 (Kumar et al, 2016).

Prediction of Protein Structure and Domain Architecture

The AAS of RELA protein was submitted i n $\mathrm{t} \quad \mathrm{ph}$ y r e 2 o n $1 \mathrm{ine}$ ( http://www.sbg.bio.ic.ac.uk /phyre $2 /$ html/page.cgi) for protein structure prediction and analysis. The protein parameters (physical and chemical properties) of pig AAS length of 551 were obtained from Expasy Bioinformatics resource portals where Protfam tool was used to obtain the protein parameters.

Domain architecture of RELA gene was predicted using SMART software online (Tables 3). The amino acids sequence obtained from NCBI database was used to determine the domain architecture.

\section{Results and discussions}

The organisms and the accession numbers of their amino acid sequence (AAS) with their sequence length that were retrieved from NCBI and Uniprot were shown in Table 1. There was high variation of AAS among the mammals where only four mammals have the same AAS length (551) with pig while others were not as shown in Table 1. The animals that have the same length of AAS with pigs were Warthog, Cattle, Wild yak and Gibbon.AAS length is the number of bases or amino acid (AA) in the canonical sequences. The mammals with different AAS from that of pigs were not bolded. The sequence variations observed were probably by deletion or insertion of some AA sequences due to convergent evolution. These changes that occur in DNA and proteins were products of evolution (Jin, 2006). The building blocks of these biological macromolecules, nucleotide bases, and amino acids form linear sequences that determine the primary structure of the molecules (Kedasher et al.,

Table 1: The percent identity and si milarity between the AAS of RELA gene of Pig and of other

\begin{tabular}{lccc}
\hline mammals & Accession number & Class of animals & $\begin{array}{c}\text { Amino acid Sequence } \\
\text { length }\end{array}$ \\
\hline Pig & F2QA75 & Mammalian & $\mathbf{5 5 1}$ \\
Warthog & F2QA76 & Mammalian & $\mathbf{5 5 1}$ \\
Bibirus & F2QA77 & Mammalian & 549 \\
Cattle & A1XG22 & Mammalian & $\mathbf{5 5 1}$ \\
Camel & TOMEL2 & Mammalian & 560 \\
Horse & F7B9X5 & Mammalian & 550 \\
Cat & A0A0D6CM2 & Mammalian & 555 \\
Giant Panda & G1MG73 & Mammalian & 528 \\
Wild Yak & L8IMF1 & Mammalian & $\mathbf{5 5 1}$ \\
Human & Q2TAM5 & Mammalian & 337 \\
Gorilla & G3SHV6 & Mammalian & 508 \\
Gibbon & G1R1D5 & Mammalian & $\mathbf{5 5 1}$ \\
Sheep & W5Q7L5 & Mammalian & 555 \\
Dog & F1PCU1 & Mammalian & 542 \\
Green monkey & A0A0D9R6K4 & Mammalian & 548 \\
Giant Panda & G1MG73 & Mammalian & 528 \\
Rat & Q7TQN4 & Mammalian & 550 \\
Mouse & Q04207 & Mammalian & 549 \\
MustelaFuro & M3YJN8 & Mammalian & 511 \\
Chinese hamster & G3IDM9 & Mammalian & 546 \\
Human & Q2TAM5 & Mammalian & 377 \\
Chimpanzee & A2T6W4 & Mammalian & 364 \\
Guinea pig & H0VZS0 & Mammalian & 496 \\
Orangutan & A2T7F9 & Mammalian & 364 \\
Chicken & A0A1D5P4Q4 & Aves & 558 \\
\hline & & &
\end{tabular}




\section{Computational Genomics of RELA gene of Pig and its protein prescription}

The gene identity and similarity between the AAS of RELA gene Pig and of other mammals is shown in Table 2.Identity can be defined as the amount of characters which match exactly between two different sequences.The RELA gene of pig shared very high percent identity with all mammals ranging from $95.5 \%$ (warthog) to $83.5 \%$ (guinea pig) with the exception pf chicken as an outgroup that had the least $(50.3 \%)$ value as shown in Table 2 . The identity percentage obtained from the alignments reveals the amount of characters that match exactly between different sequences. The percentage of
Identity observed from RELA gene of pig and the other mammals was due to high pattern of evolution and differentiation. This result also show that the RELA gene of all these mammals were homologous, perform the same function and have high conservation. According to Joshi and $\mathrm{Xu}$ (2007), the two sequences that have more than $70 \%$ of Identity have probability of more than $90 \%$ to perform the same function. This result is in corroboration with the findings of Oluwole (2017) (S100A8 gene) and that of Durosaro et al., 2016 (Bovine Reprimogene).

Table 2: The percent identity and similarity between the AAS of RELA gene of Pig and other species

\begin{tabular}{llll}
\hline Animal & Identity $(\%)$ & Similarity $(\%)$ & e-value \\
\hline Pig & 100 & 100 & 0.00 \\
Warthog & 99.5 & 99.5 & 0.00 \\
Babirus & 98.2 & 98.9 & 0.00 \\
Cattle & 93.2 & 94.6 & 0.00 \\
Camel & 95.7 & 96.7 & 0.00 \\
Horse & 94.9 & 96.4 & 0.00 \\
Cat & 93.5 & 95.1 & 0.00 \\
Giant Panda & 90.9 & 92.4 & 0.00 \\
Wild Yak & 93.0 & 94.4 & 0.00 \\
Human & 97.7 & 99.1 & 0.00 \\
Gibbon & 92.9 & 94.9 & 0.00 \\
Sheep & 91.1 & 92.7 & 0.00 \\
Dog & 91.5 & 93.8 & 0.00 \\
Green monkey & 93.0 & 94.7 & 0.00 \\
Giant panda & 90.9 & 92.4 & 0.00 \\
Rat & 89.5 & 92.0 & 0.00 \\
Mouse & 88.9 & 91.7 & 0.00 \\
Mustelafuro & 91.8 & 93.5 & 0.00 \\
Chinese hamster & 89.5 & 91.1 & 0.00 \\
Gorilla & 86.2 & 88.4 & 0.00 \\
Chimpanzee & 91.0 & 92.9 & 0.00 \\
Guinea pig & 83.5 & 85.1 & 0.00 \\
Bornean Orangutan & 89.9 & 91.3 & 0.00 \\
Chicken & 50.3 & 60.5 & 0.00 \\
\hline
\end{tabular}

The similarity percentage ranges from 85.1 to $99.5 \%$ where the Warthog had the highest percentage value while the least mammal was Guinea pig and the chicken as an out group had $60.5 \%$ as shown in Table
2. Similarity percentage can be defined as the degree of resemblance between two aligned sequences. It also reveals the extent to which residues is aligned. The higher Similarity percentage obtained between the 


\section{Oluwole}

RELA gene of pig and other species implied that they all have common ancestors and homologous since the expected-value (e-value) were low for all the species, therefore they were significant (0.05) and homologous (Lesk, 2002). They can also be used to assign protein into family.

The phylogenetic result as shown in Figure 2 , where phylogenetic relationship between RELA gene of pig and that of the other organisms was shown where an optimal tree with the sum of branch length of 0.742 was obtained. The percentage of replicate trees in which the associated taxa clustered together in the bootstrap test (1000 replicates) was shown next to the branches (Felsenstein, 1985). The tree showed that the RELA gene has two clades where the first clade contained RELA gene for the mammals while the second clade contained RELA gene for the nonmammals-Chicken (Aves) which was an out group. The RELA gene of pig was in the same cluster with the other Suidae family, Warthog and Babirusa. The close relationship observed between the RELA gene of pigs and RELA gene of other mammals implied high comparability and evolution from a most recent common ancestor. It also showed that they shared eleven common ancestors from the terminal nodes to the root of the tree. The dendrogram obtained revealed that the RELA genes of different animal were clearly separated from each other according to their classes as shown in the Figure 2.

The protein physical and chemical properties of mammals with the same AAS are shown in Table 3. Despite having the same AAS length of 551, their protein structure physical and chemical properties were not the same except only their stability (unstable) and hydropathicity (negative). The N-terminal of both the pig and warthog were both Asparagine, the cattle and gibbons were having Methionine while the Wild yak was Leucine. Pig and Warthog had almost the same physical and chemical properties as shown in the Table 3 . From the $61 \%$ residues modelled at more than $90 \%$ only $43 \%$ can be meaningfully predicted. Therefore the overall confidence was considered to be low $(<70 \%)$. The result as shown below (Figure 1) can be $\begin{array}{llllll}\mathrm{i} & \mathrm{e} & \mathrm{w} & \mathrm{e} & \mathrm{d}\end{array}$ http://www.sbg.bio.ic.ac.uk/phyre2/phyre 2_output/

From this study, the domain architecture revealed that the RELA protein contained mainly RNA Recognition motif and region of low complexity as shown in Table 4. The domainstarting positions of all the organisms range from 191 to 210 with the exception of Gorilla and orangutan that started from 6 . The termination (end) of all the proteins range from 102 to 306 amino acids. Jiang et al. (1997) reported that domain is evolutionarily more conserved than other regions of a protein and tends to evolve as units, which are gained, lost, or shuffled as one module. He also added that the identification of motifs and domains in proteins is an important aspect of the classification and functional annotation of protein sequences.

The region of low complexity start point ranged from position 173 to 462while termination positions ranged from 184 positions to 474 (Table 4). The proteins also contained region of little diversity in the sequence of amino acid composition called low complexity region. Low complexity region evolved rapidly through mitotic replication slippage and meiotic recombination event. 
Computational Genomics of RELA gene of Pig and its protein prescription

Table 3: RELA protein parameters of pig and other mammals that have the same AAS length

\begin{tabular}{llllll}
\hline $\begin{array}{l}\text { Protein } \\
\text { Parameters }\end{array}$ & Pig & Warthog & Cattle & Wild yak & Gibbon \\
\hline Carbon & 2654 & 2657 & 5322 & 2662 & 2667 \\
Hydrogen & 4175 & 4177 & 8376 & 4193 & 4190 \\
Nitrogen & 745 & 745 & 1498 & 749 & 744 \\
Oxygen & 813 & 810 & 1637 & 819 & 812 \\
Sulphur & 19 & 19 & 38 & 18 & 19 \\
Total atom & 8406 & 8408 & 16871 & 8041 & 8432 \\
Instability Index & 50.21 & 50.29 & 43.62 & 43.96 & 53.95 \\
Stability & Unstable & Unstable & Unstable & unstable & unstable \\
Aliphatic index & 72.99 & 73.18 & 72.61 & 73.14 & 73.68 \\
Hydropathicity & -0.451 & -0.450 & -0485 & -0.482 & -0.463 \\
N-terminal & Asparagine & Asparagine & Methionine & Leucine & Methionine \\
AAS =Amino acid sequence, c=carbon, $\mathrm{H}=$ hydrogen, N=nitrogen, $\mathrm{O}=$ oxygen, S= Sulphur & & \\
\hline
\end{tabular}

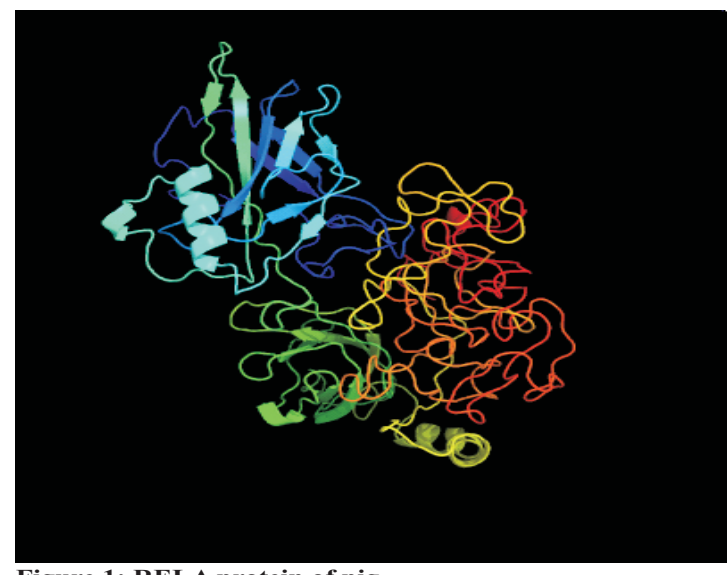

Figure 1: RELA protein of pig

Table 4 : Domain architecture of RELA using SMART

\begin{tabular}{|c|c|c|c|c|c|}
\hline \multirow{2}{*}{$\begin{array}{l}\text { Domain name } \\
\text { Organism Name }\end{array}$} & \multicolumn{3}{|c|}{ RNA Recognition motif } & \multicolumn{2}{|c|}{ Low complexity } \\
\hline & start & End & e-value & start & End \\
\hline Pig & 191 & 287 & $2.81 \mathrm{e}-22$ & 377 & 425 \\
\hline Warthog & 191 & 287 & $2.81 \mathrm{e}-22$ & 377 & 425 \\
\hline Babirusa & 191 & 287 & $1.77 \mathrm{e}-21$ & 276 & 423 \\
\hline Camels & 200 & 196 & $2.81 \mathrm{e}-22$ & 386 & 434 \\
\hline Horse & 193 & 289 & $2.81 \mathrm{e}-22$ & 324 & 346 \\
\hline Cat & 193 & 289 & $2.81 \mathrm{e}-22$ & 378 & 421 \\
\hline Cattle & 193 & 289 & $1.73 e-22$ & 395 & 402 \\
\hline Wild yak & 193 & 289 & $2.81 \mathrm{e}-22$ & 395 & 402 \\
\hline Gabbon & 193 & 289 & $2.81 \mathrm{e}-22$ & 375 & 431 \\
\hline Sheep & 193 & 289 & $2.81 \mathrm{e}-22$ & 392 & 406 \\
\hline Dog & 193 & 289 & $1.22 \mathrm{e}-22$ & 378 & 405 \\
\hline Green monkey & 210 & 306 & $2.81 \mathrm{e}-22$ & 392 & 442 \\
\hline Giant Panda & 210 & 306 & $2.81 \mathrm{e}-22$ & 396 & 424 \\
\hline Rat & 193 & 289 & $2.81 \mathrm{e}-22$ & 377 & 389 \\
\hline Chinese hamster & 194 & 290 & $2.81 \mathrm{e}-22$ & 407 & 421 \\
\hline Guinea pig & 193 & 289 & $2.81 \mathrm{e}-22$ & 462 & 474 \\
\hline Human & 193 & 289 & $2.81 \mathrm{e}-22$ & - & - \\
\hline Gorilla & 6 & 102 & $2.81 \mathrm{e}-22$ & 187 & 244 \\
\hline Orangutan & 6 & 102 & $1.7 e-22$ & 187 & 244 \\
\hline Chicken & 198 & 294 & $6.55 e-23$ & 173 & 184 \\
\hline
\end{tabular}




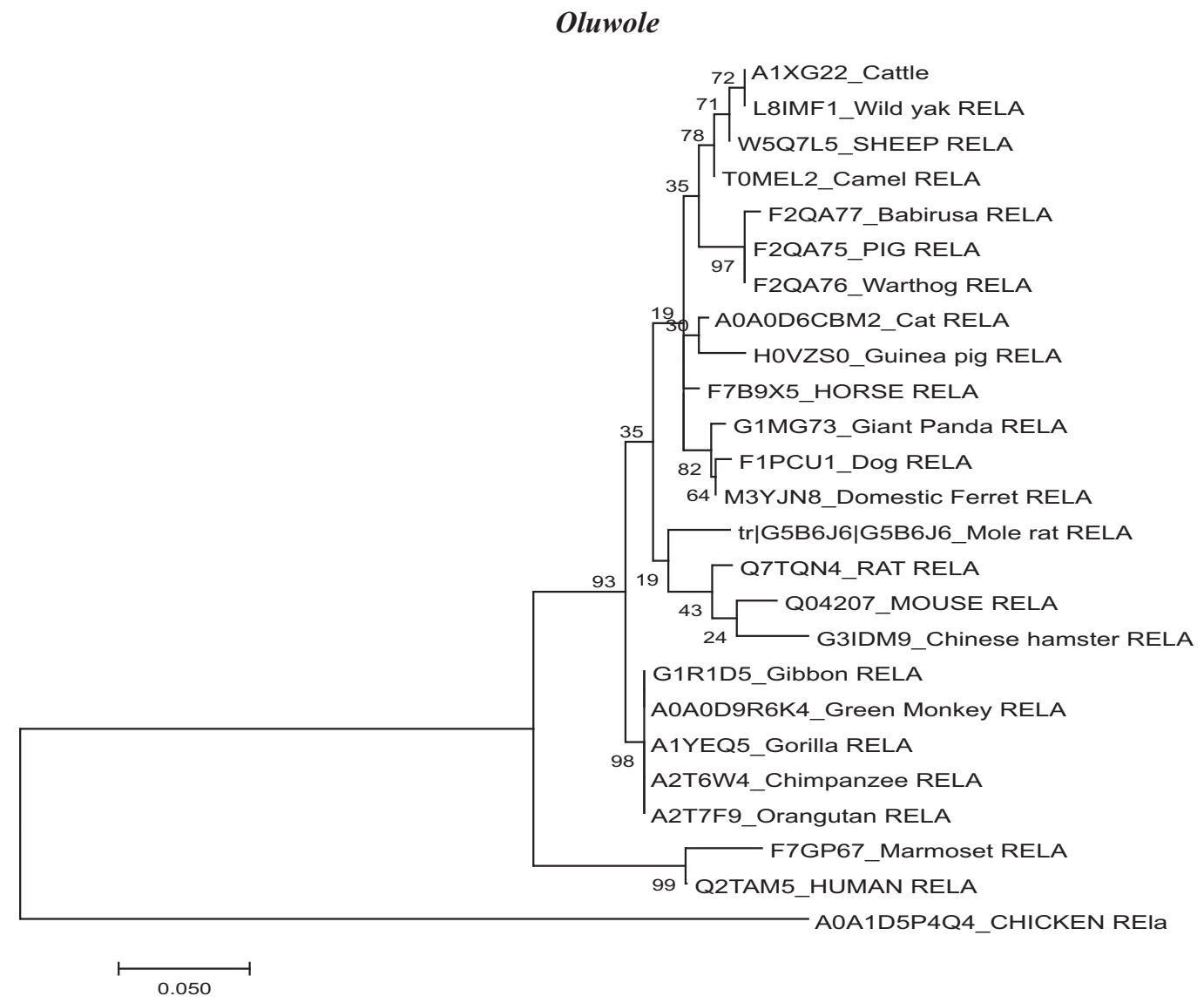

Figure 2: Phylogenetic tree of RELA gene of pig with other species

\section{Conclusion}

In conclusion, this study shows that RELA gene of pig and that of other mammalian species was highly comparable and evolved from common ancestors i.e it has high relatedness among the mammalian species studied. The high similarity and identity was observed among the RELA gene of pig and other mammalian species. The protein predicted was only $43 \%$ meaningful. The domain architecture of RELA protein structure contained mainly RNA Recognition motif and region of low complexity. The domain starting position of all the organisms ranged from 191 to 210 while the termination of all the mammals ranged from 102 to 306 amino acids. The region of low complexity starting point ranged from position 173 to 462 while termination positions ranged from 184 to 474. This study gives basic information that may be useful in the development of diseases resistance because RELA participates in adaptive immunity and responses to invading pathogens via NF- $\kappa B$ activation.

\section{References}

Baxevanis, A. D. and Quellete, B. F. F. 2001. Bioinformatics: A practical guide to Analysis of gene and protein. $2^{\text {nd }}$ edition John Wiley and sons, Inc USA. 470pp.

Bettelli, E., Dastrange M. and Oukka, M. 20005. Foxp3 interacts with nuclear factor of activated $\mathrm{T}$ cells 
and NF-kappa B to repress cytokine gene expression and effector functions of $\mathrm{T}$ helper cells. Proc. Natl. Acad. Sci. U.S.A. $102: 5138-5143$ [PubMed] [Europe PMC]

Chen, L. F. and Greene, W. C. 2004. "Shaping the nuclear action of NF-kappaB".Nature Reviews Molecular Cell Biology.5 (5): 392-401. doi: 10.1038/nrm1368. PMID 15122352

Durosaro, S. O., Ilori, B. M., Sanda, A. J. Onagbesan, O. M., Peters, S. O. and Ozoje, M. O. 2016. Mutational and evolutionary Analysis of Bovine Reprimo Gene. Nigerian Journal of animal production. The Nigerian society for animal production. Vol 43(1), page 10-19

Felsenstein J. (1985). Confidence limits on phylogenies: An approach using the bootstrap. Evolution 39:783791.

Fonseca, V. G., Fonseca, V. G., Rosa, J., Laizé, V., Gavaia, P. J. and Cancela, M. L. 2011 . Identification of a new cartilagespecific S100-like protein up$\mathrm{r}$ e g u 1 a t e d d u ri n g e $\mathrm{nd} \mathrm{o} / \mathrm{p}$ e r i c ho n d r a l mineralization in gilthead seabream. Gene Expression Patterns: GEP [2011, 11(7):448455]

GeneCards human gene database 2018 . Rela gene, protein coding and Rela oncogene. Weizmann Institute of Science. All Rights Reserved. v4.6.1 Build 19

Jiang, W., Hou Y. and Inouye, M. 1997. CspA, the major cold-shock protein of Escherichia coli, is an RNA chaperone. Journal of
Biology and Chemistry. 272:196-202.

J i n, X . 2006 . E s s e n t i a 1 Bioinformatics.Cambridge University Press. 1:3-27.

Josh, T. and Xu D. 2007. Quantitative assessment of relationship between sequence similarity and function similarity. Biomed Central Genomics. 8;222-234.

Kedersha, N. L., Gupta, M., Li, W., Miller, I. and Anderson, P. 1999. RNA-binding proteins TIA-1 and TIAR link the phosphorylation of eIF- $2 \alpha$ to the assembly of mammalian stress granules. Journal of Cellular Biology.;147: 1431-1442.

Kumar, S., Stecher, G. and Tamura, K. 2016. MEGA7: Molecular Evolutionary Genetics Analysis version 7.0 for bigger datasets.Molecular Biology and Evolution 33:1870-1874.

Lehninger, A. L., Nelson, L. D. and Cox, M. M. 1993. Principe of Biochemistry $2^{\text {nd }}$ edition. Worth publisher Inc. USA. 432pp

Lesk, A. M. 2002. Introduction to Bioinformatics $2^{\text {nd }}$ Edition. Oxford University press, Oxford, 255pp.

Li, Q. and Verma, I. M. 2002."NF-kappaB regulation in the immune system". Nature Reviews. Immunology.2 (10): 725-34. doi: $10.1038 / \mathrm{nri910}$. PMID 12360211

Nolan, G. P., Ghosh, S., Liou, H. C., Tempst, P., Baltimore, D. 1991. "DNA binding and I kappa B inhibition of the cloned p65 subunit of NF-kappa B, a relrelated polypeptide".Cell.64 (5): 961-9. doi: 10.1016/0092- 
8674(91)90320-X. PMID 2001591

Saitou, N. and Nei, M. 1987. The neighbor-joining method: A new method for reconstructing phylogenetic trees. Molecular Biology and Evolution 4:406425.Uniprot 2017. Protein $\begin{array}{lllllllll}d & a & t & a & b & a & s & e\end{array}$. http://www.uniprot.org/unipro $\mathrm{t} / \mathrm{C} 3 \mathrm{~S} 7 \mathrm{~K} 5$

Zuckerkandl E. and Pauling $L$. 1965. Evolutionary divergence and convergence in proteins. Edited in Evolving Genes and Proteins by V. Bryson and H.J. Vogel, pp. 97-166. Academic Press, New York.
Uniprot knowledgebase (UniprotKB). http://www.uniprot.org/uniprot/ Q04206

Received: $14^{\text {th }}$ November, 2017 Accepted: $18^{\text {th }}$ February, 2018 